G. ZUCCARELLO, J. G. MAYANS, I. ESCOFET, D. SCHARNAGEL, M. S. KIRILLOVA, A.

H. PÉREZ-JIMENO, P. CALLEJA, J. R. BOOTHE, A. M. ECHAVARREN* (BARCELONA

INSTITUTE OF SCIENCE AND TECHNOLOGY AND UNIVERSITAT ROVIRA I VIRGILI,

TARRAGONA, SPAIN)

Enantioselective Folding of Enynes by Gold(I) Catalysts with a Remote $C_{2}$-Chiral Element

J. Am. Chem. Soc. 2019, 141, 11858-11863.

\title{
Gold-Catalyzed Enantioselective Cyclizations of Enynes through Remote Enantioinduction
}

$(R, R)$-Au cat $(4 \mathrm{~mol} \%)$

DCE, $24^{\circ} \mathrm{C}, 12 \mathrm{~h}$ to $14 \mathrm{~d}$

17 examples

up tp $99 \%$ yield
er up to $96: 4 R^{1}=\mathrm{CO}_{2} \mathrm{Me}, \mathrm{R}^{2}=\mathrm{Me}, \mathrm{R}^{3}=\mathrm{H}, 64 \%$ yield, er $=93: 7$ $\mathrm{R}^{1}=\mathrm{CH}_{2} \mathrm{OMe}, \mathrm{R}^{2}=\mathrm{Me}, \mathrm{R}^{3}=\mathrm{OMe}, 97 \%$ yield, er $=96: 4$ $\mathrm{R}^{1}=\mathrm{CH}_{2} \mathrm{OMe}, \mathrm{R}^{2}=\mathrm{Me}, \mathrm{R}^{3}=t-\mathrm{Bu}, 99 \%$ yield, er $=91: 9$

\section{Category}

Metals in Synthesis

\section{Key words}

gold catalysis

remote

enantioinduction

enynes

carexanes

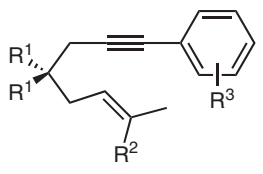

(up to $0.09 \mathrm{mmol}$ ) $\mathrm{AgPF}_{6}(4 \mathrm{~mol} \%)$
Synthesis of azabicyclo[4.1.0]hept-4-enes:<smiles>[R]C(=C)CNC#CBr</smiles>

(up to $0.094 \mathrm{mmol}$ )

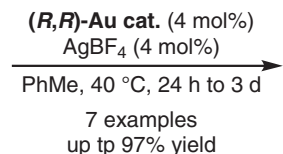

up tp $97 \%$ yield er up to $96: 4$

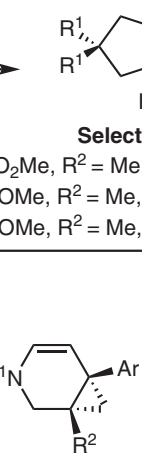

hathales:

Synthesis of 1,2-dihydronaphthalenes:

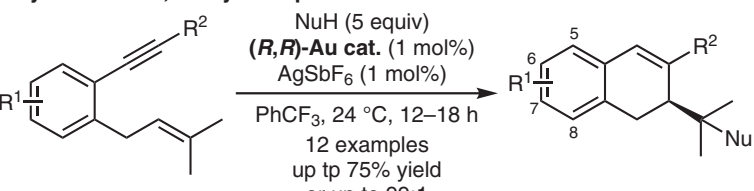
tp $75 \%$ yield

(up to $2.62 \mathrm{mmol}$ ) er up to $99: 1$

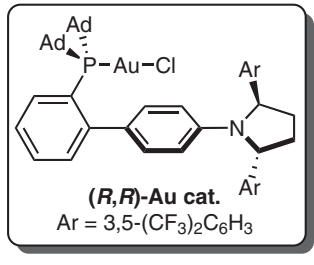

Selected examples:

$R^{1}=\mathrm{Cbz}, \mathrm{R}^{2}=\mathrm{H}, \mathrm{Ar}=\mathrm{Ph}, 89 \%$ yield, er $=90: 10$ $R^{1}=\mathrm{Ts}, \mathrm{R}^{2}=\mathrm{H}, \mathrm{Ar}=4-\mathrm{MeOC}_{6} \mathrm{H}_{4}, 74 \%$ yield, er $=94: 6$
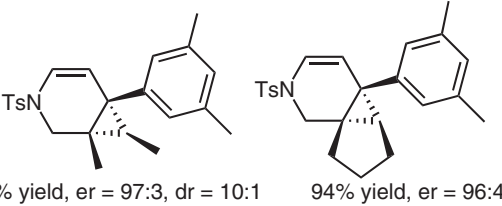

$97 \%$ yield, er $=97: 3, d r=10: 1 \quad 94 \%$ yield, er $=96: 4$

\section{Selected examples:}

$\mathrm{R}^{1}=\mathrm{H}, \mathrm{R}^{2}=\mathrm{Ph}, \mathrm{NuH}=\mathrm{BuOH}, 75 \%$ yield, er $=95: 5$ $\mathrm{R}^{1}=\mathrm{H}, \mathrm{R}^{2}=4-\mathrm{ClC}_{6} \mathrm{H}_{4}, \mathrm{NuH}=\mathrm{MeOH}, 52 \%$ yield, er $=96: 4$ $\mathrm{R}^{1}=6-\mathrm{CF}_{3}, \mathrm{R}^{2}=\mathrm{Ph}, \mathrm{NuH}=\mathrm{H}_{2} \mathrm{O}, 69 \%$ yield, er $=94: 6$ $\mathrm{R}^{1}=6-\mathrm{OMe}, \mathrm{R}^{2}=\mathrm{Ph}, \mathrm{NuH}=\mathrm{H}_{2} \mathrm{O}, 60 \%$ yield, er $=97: 3$

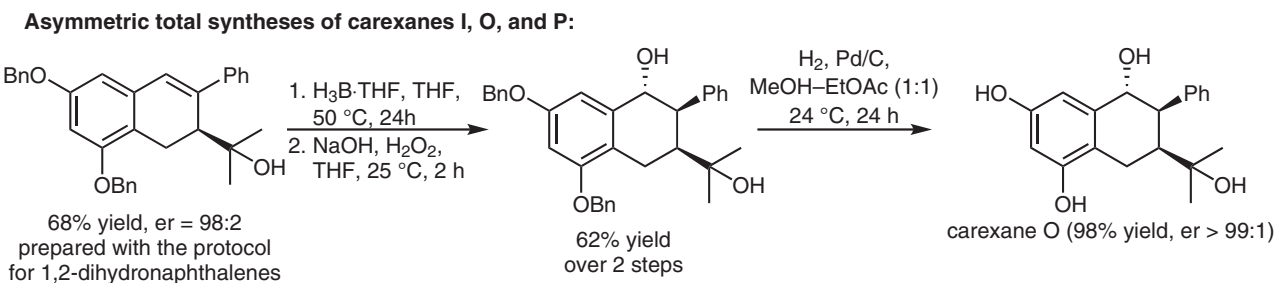

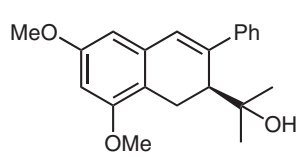

$60 \%$ yield, er $=99: 1$ prepared with the protocol for 1,2-dihydronaphthalenes

2. $\mathrm{NaOH}, \mathrm{H}_{2} \mathrm{O}_{2}$,

THF, $25^{\circ} \mathrm{C}, 2 \mathrm{~h}$ (67\% yield over 2 steps) 3. TIPSOTf, 2,6-lutidine, $\mathrm{CH}_{2} \mathrm{Cl}_{2}$ $0^{\circ} \mathrm{C}, 90 \mathrm{~min}$ 4. Burgess reagent THF, $24^{\circ} \mathrm{C}, 1 \mathrm{~h}$ (83\% yield over 2 steps)

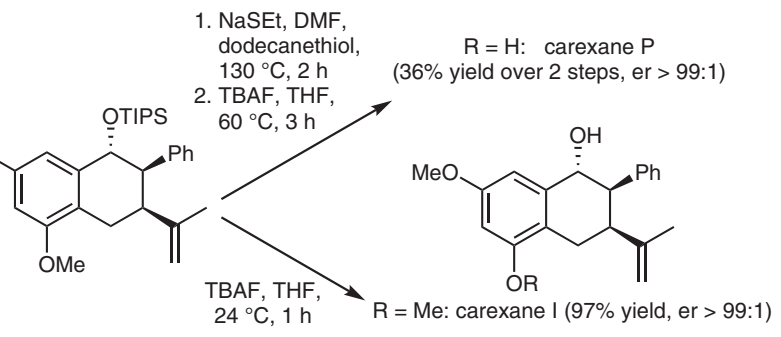

Significance: Various cyclizations were realized in an enantioselective fashion through the use of a modified JohnPhos ligand with a distal $C_{2}$ 2,5-diarrylpyrrolidine.
Comment: Hydrocyclization products were used successfully in the first enantioselective total syntheses of three natural products from the carexane family. 